\title{
CERTAIN CHARACTERIZATIONS OF VARIETIES OF BANDS
}

\author{
by J. A. GERHARD and MARIO PETRICH*
}

(Received 15th December 1986)

\section{Introduction and summary}

The lattice of varieties of bands was constructed in [1] by providing a simple system of invariants yielding a solution of the world problem for varieties of bands including a new system of inequivalent identities for these varieties. References [3] and [5] contain characterizations of varieties of bands determined by identities with up to three variables in terms of Green's relations and the functions figuring in a construction of a general band. In this construction, the band is expressed as a semilattice of rectangular bands and the multiplication is written in terms of functions among these rectangular band components and transformation semigroups on the corresponding left zero and right zero direct factors.

The present paper is essentially a continuation of [1]. We extend the abovementioned characterizations of varieties by means of Green's relations and parameters in the construction of bands to all varieties of bands. In addition, we characterize the congruence on the lattice of varieties of bands induced by the left trace relation on the corresponding fully invariant congruences.

Section 2 contains the terminology and notation and a few auxiliary results to be used later. The needed material concerning invariants and identities, established in [1], is gathered in Section 3 for easy reference. The purpose of Section 4 is establishing the main characterization theorem for the varieties of bands of the form $\left[G_{n}=H_{n}\right]$. An abbreviated version of the same type of analysis for the varieties $\left[G_{n}=I_{n}\right]$ comprises Section 5. One of the characterizations from these two sections is interpreted in Section 6 in terms of structure mappings on an arbitrary band. Left trace relation on the fully invariant congruences on a free band is transferred to the lattice of varieties of bands in Section 7 and studied in some detail.

In [1] we made a notational distinction between equality in the free semigroup and equality in the free band. In the present paper we drop this distinction.

\section{Preliminaries}

We first record here the notation to be used throughout the paper.

Notation 2.1. We will consistently use the following symbolism.

$B$-an arbitrary band.

$B^{1}-\mathrm{a}$ band $B$ with an identity adjoined.

$X$-a fixed countably infinite set. Elements of $X$ are called variables.

*Research supported by NSERC of Canada. 
$F=F(X)$-the free semigroup on $X$. Elements of $F(X)$ are called words. They are finite strings of elements of $X$ written as $x_{1} x_{2} \ldots x_{n}$ where $x_{1}, x_{2}, \ldots, x_{n} \in X$. The product is concatenation.

$F^{1}$-the identity added to $F$ to obtain $F^{1}$ is denoted by $\varnothing$. It is thought of as the empty string.

$F \mathscr{B}=F \mathscr{B}(X)$-the free band on $X$.

$c(w)$-the content of $w \in F$ is the set of variables occurring in $w$. By definition $c(\varnothing)=\varnothing$. $\#(w)$ - the number of elements in $c(w)$, that is the number of distinct variables occurring in $w$.

$\bar{w}$-the dual of $w$ is the word obtained from $w$ by reversing the order of variables.

That is, if $w=x_{1} x_{2} \ldots x_{n}$ with $x_{1}, x_{2}, \ldots, x_{n} \in X$, then $\bar{w}=x_{n} x_{n-1} \ldots x_{1}$.

Let $w=u x v$ where $c(w)=c(u x)$ and $c(w) \neq c(u)$. Then

$s(w)=u-$ the longest left cut of $w$ containing all but one of the variables of $w$. The definition is to include $s\left(x^{m}\right)=\varnothing$.

$\sigma(w)=x$ - the last variable to occur in $w$ in order from the left.

Note that $c(w)=c(s(w)) \cup\{\sigma(w)\}$.

$\varepsilon(w)=\sigma(\bar{w})$ - the last variable to occur in $w$ in order from the right.

$e(w)=\overline{s(\bar{w})}$ - the longest right cut of $w$ containing all but one of the variables of $w$.

$\left[u_{\alpha}=v_{\alpha}\right]$ - the variety of bands determined by the family of identities $u_{\alpha}=v_{\alpha}$. The defining identity for bands $x^{2}=x$ is consistently omitted.

$\mathscr{L} \mathscr{Z}=[x=x y]$ 一 the variety of left zero semigroups.

$\langle\mathscr{A}\rangle$ - the variety of bands generated by the class $\mathscr{A}$ of bands.

$u=v \Rightarrow p=q$-implication of identities.

$\vee, \wedge-$ join and meet operations on a lattice.

$[u, v]$ - the interval in a lattice $L$ bounded by $u, v \in L$.

$\mathscr{L}(\mathscr{B})$ - the lattice of varieties of bands (see Figure 1 ).

$\rho_{\mathscr{r}}$-the fully invariant congruence on $F \mathscr{B}$ determined by $\mathscr{V} \in \mathscr{L}(\mathscr{B})$. It is well known that $\mathscr{V} \rightarrow \rho_{\mathscr{V}}$ is a lattice anti-isomorphism of $\mathscr{L}(\mathscr{B})$ with the lattice of fully invariant congruences on $F \mathscr{B}$.

$\mathscr{V}_{\rho}$ - the variety of bands associated with the fully invariant congruence $\rho$ on $F(\mathscr{B})$. The map $\rho \rightarrow \mathscr{V}_{\rho}$ is the inverse of the map $\mathscr{V} \rightarrow \rho_{\mathscr{V}}$ discussed above.

$\theta$ is over $\mathscr{K}$-if $\theta$ is a congruence on a semigroup $S$ and $\mathscr{K}$ is a class of semigroups, then $\theta$ is over $\mathscr{K}$ if every idempotent $\theta$-class of $S$ belongs to $\mathscr{K}$.

$\mathscr{U} \circ \mathscr{V}$ - Let $\mathscr{U}, \mathscr{V} \in \mathscr{L}(\mathscr{B}) . \mathscr{U} \circ \mathscr{V}$ is the Malcev product of $\mathscr{U}$ and $\mathscr{V}$ defined by $\mathscr{U} \circ \mathscr{V}=$ $\{B \mid B$ a band for which there exists a congruence $\theta$ on $B$ such that $\theta$ is over $\mathscr{U}$ and $B / \theta \in \mathscr{V}\}$.

$\rho^{*}$ - the congruence generated by the relation $\rho$.

$\rho^{0}$-the largest congruence contained in the equivalence relation $\rho$.

$\pi$ - for a band $B$ written as a semilattice $Y$ of rectangular bands $B_{a}, \pi: B \rightarrow Y$ is the natural homomorphism with $\pi a=\alpha$ if $a \in B_{\alpha}$.

$D$-a reference to which a $D$ is appended means the dual of that statement.

We now establish a few simple lemmas that will be used later.

Lemma 2.2. In any band $B$, we have

$$
a \mathscr{L}^{0} b \Leftrightarrow x a=x a x b, x b=x b x a \text { for all } x \in B .
$$




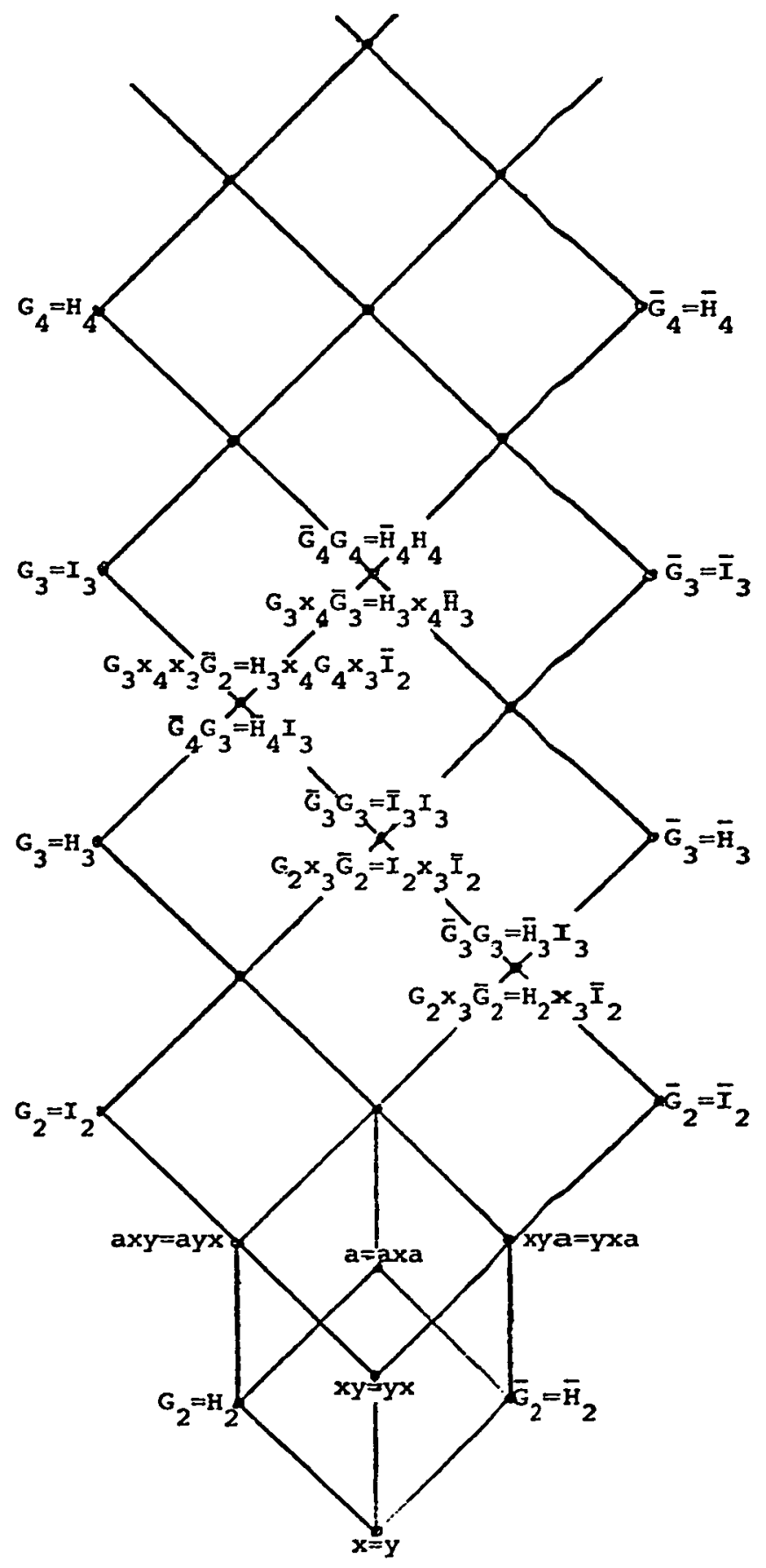

FIGURE 1 The lattice of proper varieties of bands. 
Proof. By definition, we have

$$
a \mathscr{L}^{0} b \Leftrightarrow x a=x a x b, x b=x b x a \text { for all } x \in B^{1}
$$

since $\mathscr{L}$ is a right congruence. Letting $x=a$ in $x a=x a x b$ yields $a=a b$ and similarly $x b=x b x a$ gives $b=b a$ for $x=b$. It then follows that (1) holds.

Corollary 2.3. In any band $B$, if $a \mathscr{D} b$, then

$$
a \mathscr{L}^{0} b \Leftrightarrow x a=x a x b \text { for all } x \in B .
$$

Proof. Indeed, if $a \mathscr{D} b$ and $x a=x a x b$, then $x a \mathscr{D} x b$ and thus $x b=(x b)(x a)(x b)=x b x a$. The assertion now follows from 2.2 .

Corollary 2.4. For any $u, v \in F \mathscr{B}$, we have

$$
\mathscr{L} \mathscr{Z} \circ[u=v]=[x u=x u x v, x v=x v x u] .
$$

If $c(u)=c(v)$, then $\mathscr{L} \mathscr{Z} \circ[u=v]=[x u=x u x v]$.

Proof. This follows directly from 2.2 and 2.3 and the fact that $\mathscr{L}^{\circ}$ is over $\mathscr{L} \mathscr{Z}$.

Lemma 2.5. Let $B$ be a band. Then $B / \mathscr{L}^{0}$ is a right regular band if and only if $\mathscr{L}$ is a congruence.

Proof. Necessity. By [4, Proposition 2] we get that $\mathscr{L}^{*} \subseteq \mathscr{L}^{0}$ and since the opposite inclusion holds trivially, we obtain $\mathscr{L}^{*}=\mathscr{L}^{0}$. This means that $\mathscr{L}$ is a congruence.

Sufficiency. Since $\mathscr{L}$ is a congruence, it follows that $\mathscr{L}^{0}=\mathscr{L}^{*}$. By [4, Proposition 2], $B / \mathscr{L}^{*}$ is a right regular band and thus so is $B / \mathscr{L}^{0}$.

\section{Invariants and identities}

We first list for reference certain notation and results from [1] needed in the present paper. In particular we give the definitions of the invariants and identities introduced there. Next we establish some additional lemmas which give results on invariants and identities to be used in Sections 4 and 5 .

Notation 3.1. Let $t_{n}(\varnothing)=\varnothing$ if $t \in\{h, i, \bar{h}, i\}$. Set

$h_{2}(w)$-the first variable in $w$, called the head of $w$,

$i_{2}(w)=i_{2} s(w) \sigma(w)$ - the variables of $w$ written in order of first occurrence, called the initial part of $w$,

$$
\begin{gathered}
t_{n}(w)=\overline{t_{n}(\bar{w})} \text { for } n \geqq 2 \text { and } t \in\{h, i\}, \\
t_{n}(w)=t_{n} s(w) \sigma(w) t_{n-1}(w) \quad \text { for } n \geqq 3 \text { and } t \in\{h, i\} .
\end{gathered}
$$


Notation 3.2. We define inductively three systems of words as follows:

$$
\begin{gathered}
G_{2}=x_{2} x_{1}, \quad H_{2}=x_{2}, \quad I_{2}=x_{2} x_{1} x_{2}, \\
G_{n}=x_{n} \bar{G}_{n-1}, \quad T_{n}=G_{n} x_{n} \bar{T}_{n-1} \quad \text { for } T \in\{H, I\} \text { and } n \geqq 3 .
\end{gathered}
$$

Lemma $3.3[1,3.2]$. Let $t \in\left\{h_{n} \mid n \geqq 3\right\} \cup\left\{i_{n} \mid n \geqq 2\right\}$.

(i) $c t=c$.

(ii) $s t=t s$.

(iii) $\sigma t=\sigma$.

Lemma 3.4 [1, 3.3(iii), 6.1(i)(ii), 7.2(i), 7.3(ii)]. Let $u, v, w \in F$.

(i) $t_{n}(w)=t_{n} s(w) \sigma(w) \varepsilon(w) t_{n-1} e(w) \quad\left\{\begin{array}{l}\text { for } n \geqq 3 \text { if } t=i \\ \text { for } n \geqq 4 \text { if } t=h .\end{array}\right.$

(ii) Let $n \geqq 2$.

$$
\begin{array}{ccc}
G_{n}=H_{n} \Rightarrow u=v & \text { if and only if } \quad h_{n}(u)=h_{n}(v) . \\
G_{n}=I_{n} \Rightarrow u=v & \text { if and only if } \quad i_{n}(u)=i_{n}(v) .
\end{array}
$$

(iii) If $c(u) \neq c(v), h_{2}(u)=h_{2}(v)$ and $h_{2}(u) \neq h_{2}(v)$, then

$$
[u=v]=[a x y=a y x] \wedge[a=a x a]=\left[G_{2}=H_{2}\right] .
$$

(iv) If $c(u) \neq c(v), h_{2}(u)=h_{2}(v)$ and $h_{2}(u)=\bar{h}_{2}(v)$, then

$$
[u=v]=\left[G_{2}=H_{2}\right] \vee\left[\bar{G}_{2}=\bar{H}_{2}\right]=\left[G_{2} x_{3} \bar{G}_{2}=H_{2} x_{3} \bar{H}_{2}\right]=[a=a x a] .
$$

Lemma 3.5. Let $t_{n}$ stand for $h_{n}$ if $n \geqq 4$ or $i_{n}$ if $n \geqq 3$.

(i) $\varepsilon t_{n}=\varepsilon$.

(ii) $e t_{n}=t_{n-1} e$.

Proof. In view of 3.4(i), statement 3.3(i) can be used to easily establish both (i) and (ii).

Lemma 3.6. For $w \in F, t \in\{h, i\}$ and $n \geqq 2$, we have $t_{n}^{2}(w)=t_{n}(w)$.

Proof. The proof is by induction on $\#(w)$. The result is trivial if $n=2$ and in all cases if $\#(w)=1$. The definition of $h_{3}$ means that a separate calculation is necessary in this case, although the basic method of proof is similar in all the cases $n \geqq 3$. Recall that $h_{3}(w)=h_{3} s(w) \sigma(w) h_{2}(w)$. Replacing $w$ by $h_{3}(w)$ gives 


$$
\begin{array}{rlrl}
h_{3}^{2}(w) & =h_{3} s h_{3}(w) \sigma h_{3}(w) h_{2} h_{3}(w) & \\
& =h_{3}^{2} s(w) \sigma(w) h_{2}(w) & & \text { by } 3.3(i i) \text { and (iii) and the definition of } \hbar_{2} \\
& =h_{3} s(w) \sigma(w) h_{2}(w) & & \text { by induction } \\
& =h_{3}(w) . & &
\end{array}
$$

For the remaining cases $\left(i_{n}, n \geqq 3\right.$ and $\left.h_{n}, n \geqq 4\right)$, the inductive hypothesis is that $t_{n}^{2}(u)=$ $t_{n}(u)$ for all $n$ whenever $\#(u)<\#(w)$. Then

$$
\begin{aligned}
t_{n}^{2}(w) & =t_{n} s t_{n}(w) \sigma t_{n}(w) \varepsilon t_{n}(w) \bar{t}_{n-1} e t_{n}(w) & & \text { by } 3.4(\mathrm{i}) \\
& =t_{n}^{2} s(w) \sigma(w) \varepsilon(w) t_{n-1}^{2} e(w) & & \text { by } 3.3 \text { and } 3.5 \\
& =t_{n} s(w) \sigma(w) \varepsilon(w) t_{n-1} e(w) & & \text { by induction } \\
& =t_{n}(w) . & &
\end{aligned}
$$

Lemma 3.7. Let $y_{i}, 1 \leqq i \leqq m$ be distinct variables. Then $t_{n}\left(y_{1} \ldots y_{m}\right)=y_{1} \ldots y_{m}$ where $t=h$ for $n \geqq 3$ and $t=i$ for $n \geqq 2$.

Proof. The proof is by induction on $m$. The result is trivial if $m=1$ and is also trivial for $i_{2}$. Now

$$
\begin{aligned}
h_{3}\left(y_{1} \ldots y_{m}\right) & =h_{3}\left(y_{1} \ldots y_{m-1}\right) y_{m} h_{2}\left(y_{1} \ldots y_{m}\right) & & \\
& =h_{3}\left(y_{1} \ldots y_{m-1}\right) y_{m} y_{m} & & \text { by the definition of } h_{2} \\
& =y_{1} \ldots y_{m-1} y_{m} & & \text { by induction on } m .
\end{aligned}
$$

The inductive calculation for $t=h, n \geqq 4$ and $t=i, n \geqq 3$ is

$$
\begin{array}{rlrl}
t_{n}\left(y_{1} y_{2} \ldots y_{m}\right) & =t_{n}\left(y_{1} \ldots y_{m-1}\right) y_{m} y_{1} \tau_{n-1}\left(y_{2} \ldots y_{m}\right) & & \text { by } 3.4(\mathrm{i}) \\
& =y_{1} \ldots y_{m} y_{1} \ldots y_{m} & & \text { by induction } \\
& =y_{1} \ldots y_{m} . &
\end{array}
$$

Lemma 3.8. In any band, we have

$$
\begin{array}{ll}
i_{n}\left(G_{n}\right)=i_{n}\left(I_{n}\right)=G_{n} & \text { for } n \geqq 2, \\
h_{n}\left(G_{n}\right)=h_{n}\left(H_{n}\right)=G_{n} & \text { for } n \geqq 3 .
\end{array}
$$


Proof. Since $G_{n}$ is a product of distinct variables, the results about $G_{n}$ follow immediately from 3.7. Also

$$
\begin{aligned}
i_{2}\left(I_{2}\right) & =i_{2}\left(x_{2} x_{1} x_{2}\right)=x_{2} x_{1}=G_{2}, \\
h_{3}\left(H_{3}\right) & =h_{3}\left(x_{3} x_{1} x_{2} x_{3} x_{2}\right)=h_{3}\left(x_{3} x_{1}\right) x_{2} h_{2}\left(x_{3} x_{1} x_{2} x_{3} x_{2}\right) \\
& =x_{3} x_{1} x_{2} x_{2} \quad \text { by } 3.7 \text { and the definition of } h_{2} \\
& =x_{3} x_{1} x_{2}=G_{3} .
\end{aligned}
$$

The proof now proceeds by induction on $n$. Note that $G_{n}=x_{n} \bar{G}_{n-1}=x_{n} G_{n-2} x_{n-1}$ for $n \geqq 4$ and that the statement also holds for $n=3$ if we set $G_{1}=x_{1}$. Let $t \in\{h, i\}, T \in\{H, I\}$ and $t=i$ if and only if $T=I$. The inductive calculation (for $n \geqq 3$ if $t=i$, and $n \geqq 4$ for $t=h)$ is

$$
\begin{array}{rlrl}
t_{n}\left(T_{n}\right) & =t_{n}\left(G_{n} x_{n} \bar{T}_{n-1}\right) & \\
& =t_{n}\left(x_{n} G_{n-2}\right) x_{n-1} x_{n} \bar{t}_{n-1}\left(\bar{T}_{n-1}\right) & & \text { by } 3.4(\mathrm{i}) \\
& =x_{n} G_{n-2} x_{n-1} x_{n} \bar{t}_{n-1}\left(\bar{T}_{n-1}\right) & & \text { by } 3.7 \\
& =G_{n} x_{n} \bar{T}_{n-1} & & \text { by induction } \\
& =T_{n} . & &
\end{array}
$$

\section{Characterizations of varieties $\left[G_{n}=H_{n}\right]$}

The main result here gives a three-fold characterization of the varieties $\left[G_{n}=H_{n}\right]$ for $n \geqq 2$. The first of these is expressed in terms of identities satisfied by $B / \mathscr{L}^{0}$ where $B$ is a band and $\mathscr{L}^{0}$ is the greatest congruence on $B$ contained in $\mathscr{L}$. The second is a statement concerning the satisfaction of a type of identity in terms of the invariant $h_{n}$. The third characterization is expressed by means of an implication which is then interpreted in Section 6 in terms of a condition on structure mappings in the construction of a general band.

The varieties $\left[G_{n}=H_{n}\right]$ belong to the extreme left column of Figure 1, the lattice of varieties of bands. The remaining part of that column, namely the varieties $\left[G_{n}=I_{n}\right]$, will be subjected to the same type of analysis in the next section. By duality, we may obtain the analogous characterizations for the varieties $\left[\bar{G}_{n}=\bar{H}_{n}\right]$ and $\left[\bar{G}_{n}=\bar{I}_{n}\right]$ comprising the extreme right-hand column of Figure 1 , and by intersections the remaining varieties of bands.

Besides the introductory simple proposition and the needed notation, the theorem is preceded by two lemmas. It is followed by a comparison with an existing result which it generalizes.

As a motivation for the form of the results in this section, we state the following trivial result. 
Proposition 4.1. The following conditions on a band $B$ are equivalent.

(i) B satisfies $\mathrm{G}_{2}=\mathrm{H}_{2}$.

(ii) $B / \mathscr{L}^{0}$ satisfies $x=y$.

(iii) $B$ satisfies $w=h_{2}(w)$ for any $w \in F$.

(iv) For any $a, b \in B$, we have $a=a b$.

This also represents the first step in the hierarchy of varieties satisfying various identities of the form $G_{n}=H_{n}$. We now introduce notation which is related to the symbolism used in [1] but is designed for easier manipulation.

Notation 4.2 Let $G\left(x_{2}\right)=\bar{G}\left(x_{2}\right)=x_{2}$ and for $n \geqq 3$, inductively define

$$
\begin{gathered}
\bar{G}\left(x_{2}, x_{3}, \ldots, x_{n}\right)=\overline{G\left(x_{2}, x_{3}, \ldots, x_{n}\right)}, \\
G\left(x_{2}, x_{3}, \ldots, x_{n}\right)=x_{n} \bar{G}\left(x_{2}, x_{3}, \ldots, x_{n-1}\right) .
\end{gathered}
$$

Note that $G_{n}=G\left(x_{1}, x_{2}, x_{3}, \ldots, x_{n}\right)$.

Lemma 4.3 Every band satisfies the following implication for all $n \geqq 3$ :

$$
\left.\begin{array}{l}
\pi x_{2} \geqq \pi x_{3} \geqq \cdots \geqq \pi x_{n} \\
x_{1} \mathscr{L} x_{3} \text { if } n \text { is even } \\
x_{1} \mathscr{R} x_{3} \text { if } n \text { is odd }
\end{array}\right\} \Rightarrow\left\{\begin{array}{l}
G\left(x_{2}, x_{3}, x_{4}, \ldots, x_{n}\right)=H_{n} \\
G\left(x_{2}, x_{1}, x_{4}, \ldots, x_{n}\right)=G_{n} .
\end{array}\right.
$$

Proof. The proof is by induction on $n$. For $n=3$ the hypotheses are $\pi x_{2} \geqq \pi x_{3}$ and $x_{1} \mathscr{R} x_{3}$. These give in particular that $x_{3}=x_{3} x_{1} x_{2} x_{3}$ and $x_{1}=x_{3} x_{1}$. Consequently

$$
\begin{gathered}
G\left(x_{2}, x_{3}\right)=x_{3} x_{2}=\left(x_{3} x_{1} x_{2} x_{3}\right) x_{2}=H_{3}, \\
G\left(x_{2}, x_{1}\right)=x_{1} x_{2}=\left(x_{3} x_{1}\right) x_{2}=G_{3} .
\end{gathered}
$$

If $n>3$, we assume by the dual of the inductive hypothesis that $\bar{G}\left(x_{2}, x_{3}, \ldots, x_{n-1}\right)=$ $\bar{H}_{n-1}$ and $\bar{G}\left(x_{2}, x_{1}, \ldots, x_{n-1}\right)=\bar{G}_{n-1}$. From the latter it follows that

$$
G\left(x_{2}, x_{1}, \ldots, x_{n}\right)=x_{n} \bar{G}\left(x_{2}, x_{1}, \ldots, x_{n-1}\right)=x_{n} \bar{G}_{n-1}=G_{n} .
$$

The hypotheses also imply that $x_{n}=x_{n} \bar{G}_{n-1} x_{n}$. Therefore

$$
G\left(x_{2}, x_{3}, \ldots, x_{n}\right)=x_{n} \bar{G}\left(x_{2}, x_{3}, \ldots, x_{n-1}\right)=\left(x_{n} \bar{G}_{n-1} x_{n}\right) \bar{H}_{n-1}=G_{n} x_{n} \bar{H}_{n-1}=H_{n} .
$$

Lemma 4.4. Let $u_{1}=G_{3} x_{3} x_{1}, u_{2}=x_{2}$,

$$
u_{n}= \begin{cases}G_{n} x_{n} & \text { if } n \geqq 3 \text { is odd } \\ x_{n} \bar{G}_{n} & \text { if } n \geqq 4 \text { is even }\end{cases}
$$


(i) If $n \geqq 3$ is odd, then $G\left(u_{2}, u_{3}, u_{4}, \ldots, u_{n}\right)=H_{n}$ and $G\left(u_{2}, u_{1}, u_{4}, \ldots, u_{n}\right)=G_{n}$.

(ii) If $n \geqq 4$ is even, then $G\left(\bar{u}_{2}, \bar{u}_{3}, \bar{u}_{4}, \ldots, \bar{u}_{n}\right)=H_{n}$ and $G\left(\bar{u}_{2}, \bar{u}_{1}, \bar{u}_{4}, \ldots, \bar{u}_{n}\right)=G_{n}$.

Proof. Note that 4.2. means that $\overline{G\left(u_{2}, \ldots, u_{n}\right)}=\bar{G}\left(\bar{u}_{2}, \ldots, \bar{u}_{n}\right)$. The proof is by induction on $n$. For $n=3$, we obtain

$$
\begin{gathered}
G\left(u_{2}, u_{3}\right)=u_{3} u_{2}=G_{3} x_{3} x_{2}=H_{3}, \\
G\left(u_{2}, u_{1}\right)=u_{1} u_{2}=G_{3} x_{3} x_{1} x_{2}=G_{3} .
\end{gathered}
$$

For the inductive step with $n$ even,

$$
\begin{aligned}
& G\left(\bar{u}_{2}, \bar{u}_{3}, \ldots, \bar{u}_{n}\right)=\bar{u}_{n} \bar{G}\left(\bar{u}_{2}, \bar{u}_{3}, \ldots, \bar{u}_{n-1}\right) \\
& =\bar{u}_{n} \bar{H}_{n-1} \quad \text { by the dual of the inductive hypothesis } \\
& =G_{n} x_{n} \bar{H}_{n-1}=H_{n} \text {, } \\
& G\left(\bar{u}_{2}, \bar{u}_{1}, \ldots, \bar{u}_{n}\right)=\bar{u}_{n} \bar{G}\left(\bar{u}_{2}, \bar{u}_{1}, \ldots, \bar{u}_{n-1}\right) \\
& =\bar{u}_{n} \bar{G}_{n-1} \quad \text { by the dual of the inductive hypothesis } \\
& =G_{n} x_{n} \bar{G}_{n-1}=G_{n} \text {. }
\end{aligned}
$$

The inductive step for $n$ odd is similar, relying again on the dual of the inductive hypothesis.

We are finally ready for the first principal result of the paper.

Theorem 4.5. For $n \geqq 3$, the following conditions on $a$ band $B$ are equivalent.

(i) $B$ satisfies $G_{n}=H_{n}$.

(ii) $B / \mathscr{L}^{0}$ satisfies $\begin{cases}x y a=y x a & \text { if } n=3 \\ \bar{G}_{n-1}=\bar{H}_{n-1} & \text { if } n>3 .\end{cases}$

(iii) $B$ satisfies $w=h_{n}(w)$ for any $w \in F$.

(iv) $B$ satisfies the implication: if

$\pi x_{2} \geqq \pi x_{3} \geqq \cdots \geqq \pi x_{n}, \quad x_{1} \mathscr{L} x_{3}$ if $n$ is even, $\quad x_{1} \mathscr{R} x_{3}$ if $n$ is odd, then

$$
G\left(x_{2}, x_{3}, x_{4}, \ldots, x_{n}\right)=G\left(x_{2}, x_{1}, x_{4}, \ldots, x_{n}\right)
$$

Proof. (i) implies (ii). Let $n=3$. Since $c(x y a)=c(y x a)$, it is sufficient by 2.3 to show that

$$
z(x y a)=z(x y a) z(y x a)
$$


holds in $B$. We have

$$
h_{3}(z x y a)=h_{3}(z x y) a a=h_{3}(z x y a z y x a)
$$

and therefore by 3.4(ii), (1) holds in $B$.

Let $n>3$. Then $c\left(\bar{G}_{n-1}\right)=c\left(\bar{H}_{n-1}\right)$. As above it is sufficient to show that $x_{n} \bar{G}_{n-1}=$ $x_{n} \bar{G}_{n-1} x_{n} \bar{H}_{n-1}$ holds in $B$. But this is just $G_{n}=H_{n}$.

(ii) implies (i). Let $n=3$. Then $x y a \mathscr{L}$ yxa holds in $B$ and in particular $x y a=x y a y x a$ holds in $B$. Set $x=x_{3}, y=x_{1} x_{2}, a=x_{2}$. Then $B$ satisfies

$$
x_{3} x_{1} x_{2}=x_{3} x_{1} x_{2} x_{1} x_{2} x_{3} x_{2}=x_{3} x_{1} x_{2} x_{3} x_{2}: G_{3}=H_{3} .
$$

If $n>3$, then since $B / \mathscr{L}^{0}$ satisfies $\bar{G}_{n-1}=\bar{H}_{n-1}$ it follows that $B$ in particular satisfies

$$
x_{n} \bar{G}_{n-1} x_{n} \bar{H}_{n-1}=x_{n} \bar{G}_{n-1}: H_{n}=G_{n} \text {, }
$$

as required.

(i) implies (iii). By 3.4 (ii), it is enough to show that $h_{n}(w)=h_{n} h_{n}(w)$ which is just 3.6.

(iii) implies (i). By 3.8 we have $G_{n}=h_{n}\left(H_{n}\right)=H_{n}$.

(i) implies (iv). The hypotheses of (iv) are the same as those of 4.3. It therefore follows from 4.3 and (i) that

$$
G\left(x_{2}, x_{1}, \ldots, x_{n}\right)=G_{n}=H_{n}=G\left(x_{2}, x_{3}, \ldots, x_{n}\right) .
$$

(iv) implies (i). The $u_{i}$ given in 4.4 satisfy the hypotheses of condition (iv). Consequently

$$
G_{n}=G\left(u_{2}, u_{1}, u_{4}, \ldots, u_{n}\right)=G\left(u_{2}, u_{3}, u_{4}, \ldots, u_{n}\right)=H_{n} .
$$

We now discuss the contents of 4.5 .

Comparison 4.6. For the case $n=3$, the above theorem is essentially the dual of $[5$, II.3.8] which we reproduce below. In this sense, 4.5 may be viewed as a generalization of the cited reference; for the case $n=2$, see 4.1 .

Proposition [5, II.3.8]. The following conditions on a band $S$ are equivalent.

(i) $\alpha^{(i, \mu)}=c$.

(ii) $\mathscr{R}$ is a left normal band congruence.

(iii) $S$ is a right seminormal band: $y \times a=y a y x a$.

(iv) $S$ satisfies every identity $u=v$ with $h(u)=h(v), r(u) \sim r(v)$.

(v) $S$ satisfies an identity $u=v$ with $h(u)=h(v), i(u) \neq i(v), r(u) \sim r(v)$.

The following table describes the comparison. 
$[5$, II.3.8D $]$

$$
n=3
$$

$$
\text { 4.5(iii) }
$$

$[1,7.1($ ii) $]$
Comments

in a different notation as explained in Section 6 in view of 2.5

see 4.8

see 4.9

Interpretation 4.7. In 4.5 for $n \geqq 4$,

(i) means that $\langle B\rangle \subseteq\left[G_{n}=H_{n}\right]$,

(ii) means that $\left\langle B / \mathscr{L}^{0}\right\rangle \subseteq\left[\bar{G}_{n-1}=\bar{H}_{n-1}\right]$ where the latter is in the extreme right-hand column of Figure 1 immediately below $\left[G_{n}=H_{n}\right]$.

The equivalence of (i) and (iii) means that $\left[G_{n}=H_{n}\right]=\bigcap_{w \in F}\left[w=h_{n}(w)\right]$.

Proposition 4.8. The following are equivalent for any band $B$.

(i) $w=h_{3}(w)$ holds for all $w \in F$.

(ii) $u=v$ holds for all $u, v \in F$ with $h_{2}(u)=h_{2}(v)$ and $u \mathscr{R} v$.

Proof. (i) implies (ii). Since $u \mathscr{R} v$, we have $s(u)=s(v)$ and $\sigma(u)=\sigma(v)$. Therefore

$$
\begin{aligned}
h_{3}(u) & =h_{3} s(u) \sigma(u) h_{2}(u) \\
& =h_{3} s(v) \sigma(v) h_{2}(v)=h_{3}(v) .
\end{aligned}
$$

If (i) holds, then $u=h_{3}(u)=h_{3}(v)=v$.

(ii) implies (i). The proof is by induction on $\#(w)$. For $\#(w)=1$, the result is trivial. For \#(w) $\geqq 2$,

$$
\begin{aligned}
h_{3}(w) & =h_{3} s(w) \sigma(w) h_{2}(w) & & \\
& =s(w) \sigma(w) h_{2}(w) & & \text { by induction } \\
& =w & & \text { by (ii). }
\end{aligned}
$$

Observe that 4.8 (ii) is a restatement of $[5$, II.3.8(iv) $]$ in the present notation.

Proposition 4.9. The following are equivalent for any band $B$.

(i) $B$ satisfies an identity $u=v$ with $h_{2}(u)=h_{2}(v), i_{2}(u) \neq i_{2}(v), u \mathscr{R} v$.

(ii) B satisfies an identity $u=v$ with $h_{3}(u)=h_{3}(v), i_{2}(u) \neq i_{2}(v)$.

Proof. (i) implies (ii). Since $h_{3}(u)=h_{3} s(u) \sigma(u) h_{2}(u)$, it follows that if the conditions of (i) hold, then so do the conditions of (ii).

(ii) implies (i). If $B$ satisfies $u=v$ as in (ii), then $B$ satisfies $u=u v$ and the conditions in (ii) for $u=v$ imply those of (i) for $u=u v$.

Remark that $4.9(\mathrm{i})$ is $[5,3.8(\mathrm{v}) \mathrm{D}]$ and 4.9 (ii) is $[1,7.1$ (ii)] in the present notation. 


\section{Characterizations of varieties $\left[G_{n}=I_{n}\right]$}

The analysis performed in the preceding section on the varieties $\left[G_{n}=H_{n}\right]$ can be done also for the varieties $\left[G_{n}=I_{n}\right]$. In view of strong similarity of these analyses, we restrict our attention to the differences and supply only the needed minimum of explanations.

Lemma 5.1. Every band satisfies the following implication for $n \geqq 3$

$$
\left.\begin{array}{l}
\pi x_{2} \geqq \pi x_{3} \geqq \cdots \geqq \pi x_{n} \\
x_{1} \mathscr{L} x_{2} \text { if } n \text { is odd } \\
x_{1} \mathscr{R} x_{2} \text { if } n \text { is even }
\end{array}\right\} \Rightarrow\left\{\begin{array}{l}
G\left(x_{2}, x_{3}, \ldots, x_{n}\right)=I_{n} \\
G\left(x_{1}, x_{3}, \ldots, x_{n}\right)=G_{n} .
\end{array}\right.
$$

Proof. The proof is by induction on $n$. For $n=3$ the hypotheses are $\pi x_{2} \geqq \pi x_{3}$ and $x_{1} \mathscr{L} x_{2}$. These give in particular that $x_{3}=x_{3} x_{1} x_{2} x_{3}, x_{2}=x_{2} x_{1} x_{2}$ and $x_{1}=x_{1} x_{2}$. Hence

$$
\begin{gathered}
G\left(x_{2}, x_{3}\right)=x_{3} x_{2}=\left(x_{3} x_{1} x_{2} x_{3}\right)\left(x_{2} x_{1} x_{2}\right)=I_{3}, \\
G\left(x_{1}, x_{3}\right)=x_{3} x_{1}=x_{3}\left(x_{1} x_{2}\right)=G_{3} .
\end{gathered}
$$

The inductive argument is similar to the one in the proof of 4.3 .

Lemma 5.2. Let $v_{1}=\bar{G}_{2}, v_{2}=\bar{I}_{2}\left(=I_{2}\right)$,

$$
v_{n}= \begin{cases}G_{n} x_{n} & \text { if } n \geqq 3 \text { is odd } \\ x_{n} \bar{G}_{n} & \text { if } n \geqq 4 \text { is even }\end{cases}
$$

(i) If $n \geqq 3$ is odd, then $G\left(v_{2}, v_{3}, \ldots, v_{n}\right)=I_{n}$ and $G\left(v_{1}, v_{3}, \ldots, v_{n}\right)=G_{n}$.

(ii) If $n \geqq 4$ is even, then $G\left(v_{2}, v_{3}, \ldots, v_{n}\right)=I_{n}$ and $G\left(v_{1}, v_{3}, \ldots, v_{n}\right)=G_{n}$.

Proof. Compare the proof of 4.4. In fact,

$$
\begin{aligned}
& G\left(v_{2}, v_{3}\right)=v_{3} v_{2}=G_{3} x_{3} \bar{I}_{2}=I_{3}, \\
& G\left(v_{1}, v_{3}\right)=v_{3} v_{1}=G_{3} x_{3} \bar{G}_{2}=G_{3} .
\end{aligned}
$$

The inductive part of the proof is similar to the corresponding proof in 4.4.

The desired characterization follows. 
Theorem 5.3. For $n \geqq 2$, the following conditions on a band $B$ are equivalent.

(i) B satisfies $G_{n}=I_{n}$.

(ii) $B / \mathscr{L}^{0}$ satisfies $\begin{cases}x y=y x & \text { if } n=2 \\ \bar{G}_{n-1}=\bar{I}_{n-1} & \text { if } n>2 .\end{cases}$

(iii) $B$ satisfies $w=i_{n}(w)$ for any $w \in F$.

(iv) B satisfies the implication: if $\pi x_{2} \geqq \pi x_{3} \geqq \cdots \geqq \pi x_{n}, x_{1} \mathscr{R} x_{2}$ if $n$ is even, $\quad x_{1} \mathscr{L} x_{2} \quad$ if $n$ is odd, then

$$
\begin{array}{ll}
x_{1}=x_{2} & \text { if } n=2 \\
G\left(x_{2}, x_{3}, x_{4}, \ldots, x_{n}\right)=G\left(x_{1}, x_{3}, x_{4}, \ldots, x_{n}\right) & \text { if } n \geqq 3 .
\end{array}
$$

Proof. The proof is similar to the proof of 4.5. We indicate the changes.

(i) implies (ii). For $n=2$ it is sufficient to show that

$$
z x y=z x y z y x
$$

holds in $B$. Now

$$
i_{2}(z x y)=z x y=i_{2}(z x y z y x)
$$

and therefore by $3.4($ ii), (1) holds in $B$.

For $n>2$ the proof is similar to 4.5 .

(ii) implies (i). Let $n=2$. Then $x y \mathscr{L} y x$ holds in $B$ and in particular $x y=x y y x=x y x$ holds in $B$. This is just $G_{2}=I_{2}$ with the obvious renaming of the variables. For $n>2$ compare with 4.5 .

(i) implies (iv) Compare with 4.5 and use 5.1 .

(iv) implies (i) Compare with 4.5 and use 5.2 .

As in the preceding section, a comparison with the earlier results as well as an interpretation can be given in this case. The comparison is with [5, II.3.12D] for $n=2$ and $[5,11.3 .5 \mathrm{D}]$ for $n=3$. We omit the details.

\section{Structure mappings}

Condition (iv) in 4.5 admits an interpretation in terms of structure mappings [,] figuring in the construction of an arbitrary band to be reproduced below. This structure theorem is a specialization of the structure theorem for general completely regular semigroups in [6] and can be easily deduced from [6, Corollary]. 
Theorem 6.1. Let $Y$ be a semilattice. For every $\alpha \in Y$, let $B_{\alpha}=L_{\alpha} \times R_{\alpha}$ where $L_{\alpha}$ is a left zero semigroup and $R_{\alpha}$ is a right zero semigroup. Assume that $B_{\alpha} \cap B_{\beta}=\varnothing$ if $\alpha \neq \beta$. For each $\alpha \in Y$, fix an element in $L_{\alpha} \cap R_{\alpha}$ and denote it by $\alpha$. Let

$$
\langle,\rangle: B_{\alpha} \times L_{\beta} \rightarrow L_{\beta}, \quad[,]: R_{\beta} \times B_{\alpha} \rightarrow R_{\beta},
$$

be functions defined wherever $\alpha \geqq \beta$. Assume that for all $a=(i, \lambda) \in B_{\alpha}$ and $b=(j, \mu) \in B_{\beta}$, the following conditions (i) and (ii) hold.

(i) If $(k, v) \in B_{a}$, then $\langle a, k\rangle=i$ and $[v, a]=\lambda$.

On $B=\bigcup_{\alpha \in y} B_{\alpha}$ define a multiplication $b y$

$$
a \circ b=(\langle a,\langle b, \alpha \beta\rangle\rangle,[[\alpha \beta, a], b]) .
$$

(ii) If $\gamma \leqq \alpha \beta$ and $(k, v) \in B_{\gamma}$, then

$$
\langle a,\langle b, k\rangle\rangle=\langle a \circ b, k\rangle, \quad[[v, a], b]=[v, a \circ b] .
$$

Then $B$ is a band. Conversely, every band is isomorphic to one so constructed.

We will need some more notation.

Notation 6.2. Let everything be as in 6.1. For $(i, \mu) \in B$, let

$$
\lambda(i, \mu)=i, \quad \rho(i, \mu)=\mu
$$

For $\gamma \in Y$ and $a_{i} \in B, i=1,2, \ldots, n$, inductively define

$$
\left[\gamma, a_{1}, a_{2}, \ldots, a_{n}\right]=\left[\left[\gamma, a_{1}, a_{2}, \ldots, a_{n-1}\right], a_{n}\right] .
$$

Lemma 6.3. With the notation as in 6.2 , we have

$$
\left[\gamma, a_{1}, \ldots, a_{n}\right]=\left[\gamma, a_{1} \ldots a_{n}\right]
$$

Proof. The proof is by induction on $n$. For $n=1$ the result is trivial and for $n=2$ it is just (ii) of 6.1. For $n \geqq 3$,

$$
\begin{array}{rlrl}
{\left[\gamma, a_{1}, \ldots, a_{n}\right]} & =\left[\left[\gamma, a_{1}, \ldots, a_{n-1}\right], a_{n}\right] & \\
& =\left[\left[\gamma, a_{1} \ldots a_{n-1}\right], a_{n}\right] & & \text { by induction } \\
& =\left[\gamma, a_{1} \ldots a_{n}\right] & & \text { by case } n=2 .
\end{array}
$$

The hypotheses of condition (iv) in 4.5 , in particular that $\pi x_{n} \leqq \pi x_{i}$ for $i<n$, imply that 


$$
\begin{aligned}
G\left(x_{2}, x_{3}, \ldots, x_{n}\right) & =x_{n} \bar{G}\left(x_{2}, x_{3}, \ldots, x_{n-1}\right) \\
& =\left(\lambda x_{n},\left[\rho x_{n}, \bar{G}\left(x_{2}, x_{3}, \ldots, x_{n-1}\right)\right] .\right.
\end{aligned}
$$

For $n=3$ therefore $G\left(x_{2}, x_{3}\right)=G\left(x_{2}, x_{1}\right)$ if and only if $\left[\rho x_{3}, x_{2}\right]=\left[\rho x_{1}, x_{2}\right]$. This is the same statement as (i) cited in 4.6 in a different notation.

For $n>3, G\left(x_{2}, x_{3}, x_{4}, \ldots, x_{n}\right)=G\left(x_{2}, x_{1}, x_{4}, \ldots, x_{n}\right)$ if and only if

$$
\left[\rho x_{n}, \bar{G}\left(x_{2}, x_{3}, x_{4}, \ldots, x_{n-1}\right)\right]=\left[\rho x_{n}, \bar{G}\left(x_{2}, x_{1}, x_{4}, \ldots, x_{n-1}\right)\right] .
$$

It is clear from its definition that $G$ is just the product of distinct variables each occurring once. In fact

$$
G\left(x_{2}, x_{3}, \ldots, x_{n}\right)= \begin{cases}x_{n} x_{n-2} \ldots x_{3} x_{2} x_{4} \ldots x_{n-1} & \text { for } n \text { odd } \\ x_{n} x_{n-2} \ldots x_{4} x_{2} x_{3} \ldots x_{n-1} & \text { for } n \text { even. }\end{cases}
$$

Therefore by 6.3, (1) holds if and only if (for example for $n$ even),

$$
\left[\rho x_{n}, x_{n-2}, x_{n-4}, \ldots, x_{4}, x_{2}, x_{3}, x_{5}, \ldots, x_{n-1}\right]=\left[\rho x_{n-1}, x_{n-2}, \ldots, x_{4}, x_{2}, x_{1}, x_{5}, \ldots, x_{n-1}\right]
$$

and it is this equality as an iteration of the basic structure map [,] which can be interpreted as a restriction on structure mappings on the band $B$ in $\left[G_{n}=H_{n}\right]$. The equality holds under the hypotheses of (iv) which can be visualized in the following way:

$$
\begin{aligned}
& x_{2} \\
& x_{1} \mathscr{P} x_{3} \\
& x_{4} \\
& \vdots \\
& x_{n}
\end{aligned} \quad \text { where } \mathscr{P}= \begin{cases}\mathscr{L} & \text { if } n \text { is even } \\
\mathscr{R} & \text { if } n \text { is odd } .\end{cases}
$$

In conjunction with the general structure Theorem 6.1 for bands, this provides a construction of bands in the variety $\left[G_{n}=H_{n}\right]$. The same type of analysis is valid for $\left[G_{n}=I_{n}\right]$.

\section{Left trace relation}

We express here the relation of having the same left trace for fully invariant congruences on $F \mathscr{B}$ in terms of the Malcev product $\mathscr{L} \mathscr{Z} \circ \mathscr{V}$ and of the classes of left fundamental members of the corresponding varieties. Also, we describe the bounds of the intervals of this congruence. The main result is preceded by a number of auxiliary ones providing some additional information. For a full discussion of the following notation and terminology, see [2]. 
Notation 7.1. In this section, we will use the following concepts and symbolism.

$B$ is left fundamental if $\mathscr{L}^{0}$ is the equality relation on $B$.

$\mathscr{L} \mathscr{F}$ - the class of all left fundamental bands.

$\leqq$, the relation on $B$ defined by: $e \leqq r f$ if $f e=e$.

$\rho \vee \mathscr{L}$-the join of the congruence $\rho$ with $\mathscr{L}$ in the lattice of equivalence relations on $B$. ltr $\rho=(\rho \vee \mathscr{L})^{0}$-the left trace of the congruence $\rho$.

$\mathscr{S}=[x y=y x]$-the variety of semilattices.

The following simple result will be used repeatedly.

Lemma 7.2. For any $\mathscr{V} \in \mathscr{L}(\mathscr{B})$,

$$
\mathscr{L} \mathscr{Z} \circ \mathscr{V}=\left\{B \in \mathscr{B} \mid B / \mathscr{L}^{0} \in \mathscr{V}\right\}
$$

Proof. Let $B \in \mathscr{L} \mathscr{Z} \circ \mathscr{V}$ so that there exists a congruence $\rho$ on $B$ over $\mathscr{L} \mathscr{Z}$ and such that $B / \rho \in \mathscr{V}$. But then $\rho \subseteq \mathscr{L}^{0}$ and thus $B / \mathscr{L}^{0}$ is a homomorphic image of $B / \rho$ and consequently $B / \mathscr{L}^{0} \in \mathscr{V}$. Conversely, if $B / \mathscr{L}^{0} \in \mathscr{V}$, then $\mathscr{L}^{0}$ being over $\mathscr{L} \mathscr{Z}$ it follows that $B \in \mathscr{L} \mathscr{Z} \circ \mathscr{V}$.

Lemma 7.3. The following statements are true.

(i) $\mathscr{L} \mathscr{Z} \circ[x=y]=\left[G_{2}=H_{2}\right]=\mathscr{L} \mathscr{Z} \circ\left[G_{2}=H_{2}\right]$.

(ii) $\mathscr{L} \mathscr{Z} \circ\left[\bar{G}_{2}=\bar{H}_{2}\right]=[a=a x a]=\mathscr{L} \mathscr{Z} \circ[a=a x a]$.

(iii) $\mathscr{L} \mathscr{Z} \circ[x y=y x]=\left[G_{2}=I_{2}\right]=\mathscr{L} \mathscr{Z} \circ\left[G_{2}=I_{2}\right]$.

(iv) $\mathscr{L} \mathscr{Z} \circ[x y a=y x a]=\left[G_{3}=H_{3}\right]=\mathscr{L} \mathscr{Z} \circ\left[G_{3}=H_{3}\right]$.

(v) $\mathscr{L} \mathscr{Z} \circ\left[\bar{G}_{n-1}=\bar{I}_{n-1}\right]=\left[G_{n}=I_{n}\right]=\mathscr{L} \mathscr{Z} \circ\left[G_{n}=I_{n}\right]$ for $n \geqq 3$.

(vi) $\mathscr{L} \mathscr{Z} \circ\left[\bar{G}_{n-1}=\bar{H}_{n-1}\right]=\left[G_{n}=H_{n}\right]=\mathscr{L} \mathscr{Z} \circ\left[G_{n}=H_{n}\right]$ for $n \geqq 4$.

Proof. Each item is of the form $\mathscr{A}=\mathscr{B}=\mathscr{C}$. The equality $\mathscr{A}=\mathscr{B}$ follows from 7.2 using the appropriate result in $4.1,4.5$ and 5.3. The inclusion $\mathscr{B} \subseteq \mathscr{C}$ is trivial. The inclusion $\mathscr{C} \subseteq \mathscr{B}$ will follow by 2.4 if we find, for each of the varieties $[u=v]$, an equation of the form $t u=t u t v$ which implies $u=v$.

(i) $t x=t x t x y \Rightarrow x=x y$ by $3.4($ iii).

(ii) ta $=$ tataxa $\Rightarrow a=a x a$ by 3.4(iv).

(iii)-(vi) Now $x_{n} G_{n}=G_{n}, x_{n} H_{n}=H_{n}, x_{n} I_{n}=I_{n}$. We prove (v); the others are similar. Indeed, $x_{n} G_{n}=x_{n} G_{n} x_{n} H_{n}$ is the identity $G_{n}=H_{n}$ and the desired implication is trivial.

Observe that the above lemma gives a precise description of the Malcev product $\mathscr{L} \mathscr{Z} \circ \mathscr{V}$ for any $\mathscr{V} \in \mathscr{L}(\mathscr{B})$.

Lemma 7.4. Let $\theta$ be a relation on a semigroup $S$. If $\theta$ is invariant under all endomorphisms of $S$, then $\theta^{*}$ is a fully invariant congruence on $S$. 
Proof. This follows by a straightforward argument from the construction of the congruence $\theta^{*}$.

Lemma 7.5. For $\mathscr{V} \in \mathscr{L}(\mathscr{B})$, we have

$$
\left(\rho_{\mathscr{r}} \cap \leqq_{r}\right)^{*}=\rho_{\mathscr{L} \mathscr{Y} \cdot \mathscr{r}}
$$

Proof. First note that $\leqq$, is a relation on $F \mathscr{B}$ which is invariant under all endomorphisms of $F \mathscr{B}$. For any fully invariant congruence $\rho$ on $F \mathscr{B}$, we then get that $\theta=\rho \cap \leqq \mathrm{r}$ is invariant under all endomorphisms and hence by $7.4, \theta^{*}$ is a fully invariant congruence on $F \mathscr{B}$. We will use this in the remainder of the proof.

The four cases $\mathscr{S} \nsubseteq \mathscr{V}$ are handled separately and then the result established for $\mathscr{S} \subseteq \mathscr{V}$. By 7.3(i) and (ii) and monotonicity for $\mathscr{S} \nsubseteq \mathscr{V}$ we need only consider the two cases (i) $\mathscr{V}=[x=y]$ and (ii) $\mathscr{V}=\left[G_{2}=H_{2}\right]$.

(i) Let $\theta=\rho_{[x=y]} \cap \leqq_{r}$. Then $\theta=\leqq_{r}=\{(p, q) \mid p=q p\}$. In particular $h_{2}(p)=h_{2}(q)$ for all $(p, q) \in \theta$. It follows from 3.4(ii) that $G_{2}=H_{2} \Rightarrow p=q$ for all $(p, q) \in \theta$. It is easy to check that $\left(G_{2}, H_{2}\right) \in \theta$. Together these two results show that $\theta^{*}=\rho_{\left[G_{2}=H_{2}\right]}$. That $\theta^{*}=\rho_{\mathscr{L} \mathscr{I} \cdot[x=y]}$ then follows from 7.3(i).

(ii) The proof is similar to (i). Indeed, let

$$
\theta=\rho_{\left[\bar{G}_{2}=\bar{H}_{2}\right]} \cap \leqq,=\left\{(p, q) \mid h_{2}(p)=h_{2}(q), p=q p\right\}
$$

Then by 3.4(iv), $a=a x a \Rightarrow p=q$ for all $(p, q) \in \theta$ and it is easy to check that $(a, a x a) \in \theta$. Therefore, using $7.3(\mathrm{ii})$, we get

$$
\theta^{*}=\rho_{[a=a \times a]}=\rho_{\mathscr{L} \mathscr{Z} \cdot[a=a \times a]}
$$

Now assume $\mathscr{S} \subseteq \mathscr{V}=[u=v]$. A proof along the lines of (i) and (ii) above can be given using 7.3 in the several cases. We prefer a more general approach. Since $\mathscr{S} \subseteq \mathscr{V}$ we have $c(u)=c(v)$ and $c(p)=c(q)$ ror all $(p, q) \in \rho_{\curlyvee}$. It follows from $[1,2.5 \mathrm{D}]$ that $x u=$ $x u x v \Rightarrow p=p q$. If $(p, q) \in \leqq_{r}$, then $p=q p$. These two results, together with the fact that $c(p)=c(q)$, give

$$
x u=x u x v \Rightarrow p=q p p q=q .
$$

By 2.4 therefore $\rho_{\mathscr{L} \mathscr{T} \cdot \vartheta} \supseteq\left(\rho_{\curlyvee} \cap \leqq_{r}\right)^{*}$. It is obvious that $(x u, x u x v) \in \rho_{\curlyvee} \cap \leqq_{r}$ which gives the other inclusion.

Lemma 7.6. For any band $B, B / \mathscr{L}^{0}$ is left fundamental.

Proof. Let $\bar{B}=B / \mathscr{L}^{0}$ and let $a, b \in B$ be such that $\bar{a} \mathscr{L}^{0} \bar{b}$. Then $\bar{x} \bar{a} \mathscr{L} \bar{x} \bar{b}$ for all $x \in B^{1}$ and thus $\bar{x} \bar{a}=\bar{x} \bar{a} \bar{x} \bar{b}$ and $\bar{x} \bar{b}=\bar{x} \bar{x} \bar{x} \bar{a}$ for all $x \in B^{1}$. It follows that

$$
x a \mathscr{L}^{0} x a x b, \quad x b \mathscr{L}^{0} x b x a \quad\left(x \in B^{1}\right)
$$


and therefore

$$
x a \mathscr{L} x a x b, \quad x b \mathscr{L} x b x a \quad\left(x \in B^{1}\right)
$$

Consequently

$$
x a=x a x b, \quad x b=x b x a \quad\left(x \in B^{1}\right)
$$

so that $a \mathscr{L}^{0} b$ and thus $a=b$. Therefore $B / \mathscr{L}^{0}$ is left fundamental.

Lemma 7.7. Let $\mathscr{U}, \mathscr{V} \in \mathscr{L}(\mathscr{R})$.

(i) $\mathscr{L} \mathscr{Z} \circ\langle\mathscr{V} \cap \mathscr{L} \mathscr{F}\rangle=\mathscr{L} \mathscr{Z} \circ \mathscr{V}$.

(ii) $\mathscr{L} \mathscr{Z} \circ \mathscr{U}=\mathscr{L} \mathscr{Z} \circ \mathscr{V} \Rightarrow \mathscr{U} \cap \mathscr{L} \mathscr{F} \subseteq \mathscr{V}$.

Proof. (i) To establish the non-trivial inclusion let $B \in \mathscr{L} \mathscr{Z} \circ \mathscr{V}$. Then $B / \mathscr{L}^{0} \in \mathscr{V}$ by 7.2 and $B / \mathscr{L}^{0} \in \mathscr{L} \mathscr{F}$ by 7.6. Therefore $B / \mathscr{L}^{0} \in\langle\mathscr{V} \cap \mathscr{L} \mathscr{F}\rangle$. Finally by 7.2 again $B \in \mathscr{L} \mathscr{Z} \circ\langle\mathscr{V} \cap \mathscr{L} \mathscr{F}\rangle$.

(ii) Let $B \in \mathscr{U} \cap \mathscr{L} \mathscr{F}$. Then $B \in \mathscr{L} \mathscr{Z} \circ \mathscr{U}=\mathscr{L} \mathscr{Z} \circ \mathscr{V}$ and hence $B=B / \mathscr{L}^{\circ} \in \mathscr{V}$ by 7.2.

We will need the following general result.

Lemma 7.8 [2, 4.7D and 4.12D]. The relation ltr on the lattice $L$ of congruences on $B$ defined by

$$
\lambda \operatorname{ltr} \rho \Leftrightarrow \operatorname{lt} \lambda=\operatorname{ltr} \rho
$$

is a complete congruence on $L$. The class of Itr containing $\rho$ is the interval

$$
\left[\left(\rho \cap \leqq_{r}\right)^{*},(\rho \vee \mathscr{L})^{0}\right]
$$

If we take $F \mathscr{B}$, the free band on a countably infinite set of generators, for $B$ in 7.8 , then the left trace restricted to the lattice of fully invariant congruences on $F \mathscr{B}$ induces a relation on $\mathscr{L}(\mathscr{B})$. This relation may again be called the left trace relation and denoted by ltr. We thus arrive at the main result of this section.

Theorem 7.9. For $\mathscr{U}, \mathscr{V} \in \mathscr{L}(\mathscr{B})$, we have

$$
\mathscr{U} \operatorname{ltr} \mathscr{V} \Leftrightarrow \mathscr{U} \cap \mathscr{L} \mathscr{F}=\mathscr{V} \cap \mathscr{L} \mathscr{F} \Leftrightarrow \mathscr{L} \mathscr{Z} \circ \mathscr{U}=\mathscr{L} \mathscr{Z} \circ \mathscr{V}
$$

For any $\mathscr{V} \in \mathscr{L}(\mathscr{B})$, the ltr-class containing $\mathscr{V}$ equals the interval $[\langle\mathscr{V} \cap \mathscr{L} \mathscr{F}\rangle, \mathscr{L} \mathscr{Z} \circ \mathscr{V}]$.

Proof. It follows from 7.5 and 7.8 that $\mathscr{L} \mathscr{Z} \circ \mathscr{V}$ is the upper bound of the ltr-class containing $\mathscr{V}$. By 7.7(i), we have that $\langle\mathscr{V} \cap \mathscr{L} \mathscr{F}\rangle$ ltr $\mathscr{V}$ and by 7.7(ii) that $\langle\mathscr{V} \cap \mathscr{L} \mathscr{F}\rangle$ is the lower bound of the ltr-class containing $\mathscr{V}$. This proves the assertion concerning the interval. If $\mathscr{U} \cap \mathscr{L} \mathscr{F}=\mathscr{V} \cap \mathscr{L} \mathscr{F}$, then $\langle\mathscr{U} \cap \mathscr{L} \mathscr{F}\rangle=\langle\mathscr{V} \cap \mathscr{L} \mathscr{F}\rangle$ and thus $\mathscr{U} \operatorname{lt} \mathscr{V}$. Conversely, if $\mathscr{U} \operatorname{ltr} \mathscr{V}$, then $\mathscr{L} \mathscr{Z} \circ \mathscr{U}=\mathscr{L} \mathscr{Z} \circ \mathscr{V}$ which by $7.7($ ii) implies that $\mathscr{U} \cap \mathscr{L} \mathscr{F}=$ 
$\mathscr{V} \cap \mathscr{L} \mathscr{F}$. This establishes the first equivalence in the statement of the theorem; the second follows from the statements already established.

As a consequence of the theorem, we obtain the precise form of the Itr-classes on $\mathscr{L}(\mathscr{B})$ as follows.

Corollary 7.10. The following intervals constitute the complete set of left trace classes on $\mathscr{L}(\mathscr{B})$.

(i) $\left[[x=y],\left[G_{2}=H_{2}\right]\right]$.

(ii) $\left[\left[\bar{G}_{2}=\bar{H}_{2}\right],[a=a x a]\right]$.

(iii) $\left[[x y=y x],\left[G_{2}=I_{2}\right]\right]$.

(iv) $\left[[x y a=y x a]\right.$, $\left.\left[G_{3}=H_{3}\right]\right]$.

(v) $\left[\left[\bar{G}_{n-1}=\bar{I}_{n-1}\right],\left[G_{n}=I_{n}\right]\right]$ for $n \geqq 3$.

(vi) $\left[\left[\bar{G}_{n-1}=\bar{H}_{n-1}\right],\left[G_{n}=H_{n}\right]\right]$ for $n \geqq 4$.

Proof. This follows directly from 7.3 and 7.9.

\section{REFERENCES}

1. J. A. Gerhard and M. Petrich, Varieties of bands revisited, submitted.

2. F. Pastijn and M. Petrich, Congruences on regular semigroups, Trans. Amer. Math. Soc. 295 (1986), 607-633.

3. M. Petrich, A construction and a classification of bands, Math. Nachr. 48 (1971), 263-274.

4. M. Petrich, Structure of completely regular semigroups, Trans. Amer. Math. Soc. 189 (1974), 211-236.

5. M. Petrich, Lectures in Semigroups (Akademie-Verlag, Berlin, 1977).

6. M. Petrich, A structure theorem for completely regular semigroups, Proc. Amer. Math. Soc. 99, (1987), 617-622.

Department of Mathematics

UNIVERSITY OF MANITOBA

Manitoba

Canada 